\title{
Trochleoplasty procedures show complication rates similar to other patellar-stabilizing procedures
}

\author{
Jordy D. P. van Sambeeck ${ }^{1}$ - Sebastiaan A. W. van de Groes ${ }^{1} \cdot$ Nico Verdonschot $^{1} \cdot$ Gerjon Hannink ${ }^{1}$
}

Received: 24 January 2017 / Accepted: 23 October 2017 / Published online: 5 December 2017

(c) The Author(s) 2017. This article is an open access publication

\begin{abstract}
Purpose Trochleoplasty aims to restore patellar stability. Various techniques have been described and almost all authors report successful results. However, the procedure has a significant risk of complications. Purpose of this study was to perform a systematic review and meta-analysis of the available literature to assess the rate of complications after the various techniques used for trochleoplasty procedures.

Materials and methods MEDLINE, EMBASE, Web of Science and Cochrane Library databases were searched. Studies on patients with recurrent patellar instability treated with a trochleoplasty with or without additional procedure, and reported complications were included. The primary outcome was the rate of complications per technique. A meta-analysis was performed whenever three or more studies per surgical technique could be included.

Results The selection process resulted in 20 studies included for analysis. A lateral facet elevating trochlear osteotomy was reported by two studies, ten studies reported on a Bereiter trochleoplasty, five on a Dejour trochleoplasty, one on an arthroscopic technique, one on a 'modified' technique and one on a recession wedge trochleoplasty. Meta-analysis showed that proportion of recurrent dislocation was 0.04 (95\% CI 0.02-0.07) for Bereiter trochleoplasty and 0.02 (95\% CI 0-0.08) for Dejour trochleoplasty. These proportions were 0.06 (95\% CI 0.02-0.13) and 0.09 (95\% CI 0.03-0.27) for recurrent instability, 0.07 (95\% CI 0.02-0.19) and 0.12 (95\% CI 0.00-0.91) for patellofemoral osteoarthritis and 0.08 (95\% CI 0.04-0.14) and 0.20 (95\% CI $0.11-0.32$ ) for further surgery respectively.

Conclusion This study demonstrates that the complications after a Bereiter and Dejour trochleoplasty including additional procedures are in the range of those of other patellar stabilizing procedures. For four other techniques, no meta-analysis could be performed. The clinical relevance of this study is that it provides clinicians with the best currently available evidence on the rate of complications after trochleoplasty procedures. This can be helpful in the process of deciding whether or not to perform such a procedure, and can be used to better inform patients about the advantages and disadvantages of different trochleoplasty procedures.
\end{abstract}

Level of evidence Level IV.

Keywords Patellofemoral instability $\cdot$ Trochlear dysplasia $\cdot$ Trochleoplasty $\cdot$ Trochlear osteotomy $\cdot$ Complications

Jordy D. P. van Sambeeck

jordy.vansambeeck@radboudumc.nl

Sebastiaan A. W. van de Groes

sebastiaan.vandegroes@radboudumc.nl

Nico Verdonschot

nico.verdonschot@radboudumc.nl

Gerjon Hannink

gerjon.hannink@radboudumc.nl

1 Department of Orthopaedics, Radboud University Medical Center, PO Box 9101, 6500 HB Nijmegen, The Netherlands

$\begin{array}{ll}\text { Abbreviations } \\ \text { CPM } & \text { Continuous passive motion } \\ \text { DVT } & \text { Deep venous thrombosis } \\ \text { MPFL } & \text { Medial patellofemoral ligament } \\ \text { MRI } & \text { Magnetic resonance imaging } \\ \text { OR } & \text { Operating room } \\ \text { PE } & \text { Pulmonary embolism } \\ \text { PF OA } & \text { Patellofemoral osteoarthritis } \\ \text { PRISMA } & \text { Preferred reporting items for systematic } \\ & \text { reviews and meta-analyses } \\ \text { ROM } & \text { Range of motion } \\ \text { VMO } & \text { Vastus medialis obliquus }\end{array}$




\section{Introduction}

Patellar dislocation occurs when the patella completely disengages from the trochlear groove. The most common recurrent symptom after patellar dislocation is patellar instability, which includes both patellar dislocation and subluxation [31]. Trochlear dysplasia has been identified as the most consistent anatomic factor present in patients with recurrent patellar dislocations [24].

Trochleoplasty is a surgical procedure designed to reshape the trochlea in patients with recurrent patellar dislocation and trochlear dysplasia. Trochleoplasty involves working directly on the patellofemoral joint, modifying the congruency between the two articulating bones and alteration of joint kinematics, with a high risk of cartilage damage. The number of trochleoplasty procedures as a primary or revision surgical treatment option in patients with recurrent patellar dislocation and trochlear dysplasia has increased over the last decade [25]. Most authors agree that trochleoplasty procedures should always be combined with soft-tissue and/or with bony procedures (e.g. medial patellofemoral ligament (MPFL) reconstruction (lowest rate of recurrence with double-limb graft configuration [37]), tibial tubercle transposition) as indicated. Therefore, a trochleoplasty procedure could be defined as a trochleoplasty including any additional stabilizing procedure.

Various techniques for trochleoplasty have been described in the past decades. Four basic trochleoplasty procedures can be distinguished: (1) the lateral-facet elevating trochleoplasty as first described by Albee [1], (2) the sulcus-deepening trochleoplasty which was first proposed by Masse [18] and later modified by Dejour [11], (3) the 'Bereiter' or 'thin-flap' procedure [6], and (4) the 'recession' or 'recession-wedge' trochleoplasty [15]. Although, the outcome measures vary widely between individual studies (e.g. Kujala Knee Score, Lysholm Score, Knee Injury and Osteoarthritis Outcome Score, etc.), most articles present satisfactory results of trochleoplasty procedures in creating a stable patellofemoral joint in terms of recurrence of patellar dislocation. However, complications are often not included as primary outcome measure but are only briefly described within the "Results" or "Discussion" in general terms. Patellar redislocation as a complication is rarely reported [25], however postoperative stiffness and return to the operating room for any reason are relatively frequent reported complications.

Trochleoplasty is a highly complex surgical technique with a significant risk for complications $[17,25$, 36]. Therefore, it is important to gain more knowledge on complications after trochleoplasty procedures. To assess the rate of complications after the various techniques used for trochleoplasty procedures a systematic review and meta-analysis of the available literature was performed. The results of this study provide clinicians with the best currently available evidence on the rate of complications after a trochleoplasty procedure. This can be helpful to properly inform the patient and to make a well-informed decision as to whether or not to perform this procedure.

\section{Materials and methods}

A systematic review was conducted and reported in accordance with the reporting guidance provided in the Preferred Reporting Items for Systematic Reviews and Meta-Analyses (PRISMA) statement [21]. The protocol was prospectively registered in PROSPERO (https://www.crd.york.ac.uk/ PROSPERO/display_record.asp?ID=CRD42015029815).

\section{Search}

MEDLINE, EMBASE, Web of Science and Cochrane Library databases were searched (last search performed 10 May 2016). The search strategy was determined in collaboration with an information specialist from the medical library of the Radboud University Medical Center. Keywords used to develop our search strategy were 'patellar instability', 'trochleoplasty', and 'complications'. The detailed search strategy is provided in "Appendix". Reference lists of included studies and relevant reviews were screened for relevant studies. No Grey literature search was undertaken.

\section{Eligibility and Study selection}

All articles were screened based on title and abstract by two reviewers (JvS, SvdG). In this screening stage, studies were excluded if they fulfilled 1 of the following criteria: (1) no trochleoplasty performed; (2) no clinical outcome study on humans (observational and/or experimental) or description of operative technique; (3) animal study, case report, review article, cadaveric study, in vitro study, biomechanical study or conference proceeding; (4) article not in English, Dutch, French, or German (all languages were screened); (5) article published before 1990. In the subsequent full text screening stage studies were further evaluated for eligibility. Studies were excluded if they met any of criteria 1-5 or 1 of the following: (6) no report of complications; (7) indication for trochleoplasty was not recurrent patellar instability. In addition, studies were excluded if they contained data also published in another included paper. In case of a study being part of a larger, original study, the original study was included. In case of reported preliminary data the most extended paper was included in the analysis. Discrepancies between the reviewers were resolved by discussion and consensus. 
The primary outcome was the rate of complications of trochleoplasty procedures. Complications were defined as: a negative outcome including returning to the operating room (OR), symptomatic hardware, loss of range of motion (ROM), increased pain/apprehension leading to return to the OR, patella redislocation/subluxation/ instability, accelerated (radiological) progression of patellofemoral osteoarthritis (PF OA), deep venous thrombosis (DVT), infection, distal femoral fracture. Complications were subdivided in minor or major complications. Minor complications included complaints of recurrence of maltracking or subluxation, loss of up to $20^{\circ} \mathrm{ROM}$ not requiring surgical treatment, increase in $\mathrm{PF} \mathrm{OA}$ to grade 2 or 3 according to Iwano classification, superficial wound infection, anesthetic complications. Major complications included redislocation of the patella, return to OR due to increase in pain or recurrence of instability or any other cause, reduced ROM requiring arthrolysis, hardware removal because of pain or crepitus, progression to grade 4 PF OA, venous thrombotic event. Residual pain, swelling or crepitus not leading to OR were considered outcomes of the procedure and not complications.

\section{Data collection and analysis}

Data were extracted from the included articles by two reviewers (JvS, SvdG) and included: study ID, number of patients, number of knees, type of trochlear dysplasia, duration of symptoms, indication for surgery, mean patient age at surgery, patient sex, previous surgery on the involved knee, type of trochleoplasty performed, additional procedures performed, type and rate of complications and (if mentioned) time when complication occurred, length of follow-up and patients lost to follow-up. In studies that reported only percentages of complications and no absolute numbers, absolute numbers of complications were calculated based on the number of patients or surgical procedures reported. Subsequently, a meta-analysis was performed whenever three or more studies per surgical technique that reported on a type of complication could be included. Despite anticipated heterogeneity, the individual study proportions were pooled. Pooled estimates of proportions with their corresponding 95\% confidence intervals were calculated using Freeman-Tukey double arcsine transformation within a random effects model framework. Heterogeneity of combined study results was assessed by $I^{2}$, and its connected Chi-square test for heterogeneity, and the corresponding $95 \%$ confidence intervals were calculated. Restricted maximum likelihood was used to estimate the heterogeneity variance. Statistical analyses were performed using $\mathrm{R}$ version 3.4.0 (R Foundation for Statistical Computing, Vienna, Austria) with package 'meta'.

\section{Quality assessment}

Quality assessment was not performed as the included articles were retrospective or prospective single-arm cohort studies and no validated scores for the methodological quality of these type of studies are available.

\section{Results}

The search strategy retrieved 1,848 unique records. Subsequent selection procedure resulted in 55 eligible articles of which 20 studies could be included in this systematic review (Fig. 1).

Table 1 displays study characteristics including population description, type of trochleoplasty performed, additional procedures performed, and the number of complications.

Trochleoplasty procedures were performed on 822 knees in 739 patients. Average age of the patients was 22.6 years (range 12-53 years). Sixty-seven percent of patients were female. Mean follow-up was 57 months, mean follow-up in individual studies ranged from 12 months to 183 months (16 studies reported mean, 2 medians, 1 range and 1 a minimum of 1 year).

Indications for trochleoplasty were recurrent patellar instability, defined as at least two patellar dislocations (in 1 study based on one documented patellar dislocation [19]), with underlying trochlear dysplasia. Ten studies $[5,7,9,14$, $19,24,28,29,33,38]$ reported trochlear dysplasia defined according to the Dejour classification of trochlear dysplasia [10] on conventional X-rays or MRI. In two studies, an elevated trochlear boss height on X-ray was additionally required as indication $[19,38]$. For some studies, indication was also based on presence of the apprehension sign or lateral patellar glide test [5, 7, 14, 24, 29, 35].

All studies reported that additional procedures were performed, except the one of Bereiter [6] that did not report on additional procedures. On average, $46 \%$ of patients had undergone previous procedures before trochleoplasty including modified Fulkerson-Elmsie Trillat osteotomy, diagnostic arthroscopy, arthroscopic/open lateral release, tibial tubercle transfer, VMO-plasty, Roux-Goldthwaite procedure and chondroplasty.

A total of 190 complications occurred in 822 knees, including recurrence of instability (subluxation and dislocation), loss of knee range of motion, development or progression of PF OA, return to OR and miscellaneous surgical complications, such as wound complications.

A lateral facet elevating trochlear osteotomy was reported by two studies $[3,16]$. A deepening trochleoplasty was reported by 17 studies: ten reported on a (modified) Bereiter trochleoplasty $[5,6,8,14,20,22,23,29,34,35]$, five on a (modified) Dejour trochleoplasty [9, 12, 19, 24, 28], one on 
Fig. 1 PRISMA flow diagram

\section{PRISMA 2009 Flow Diagram}

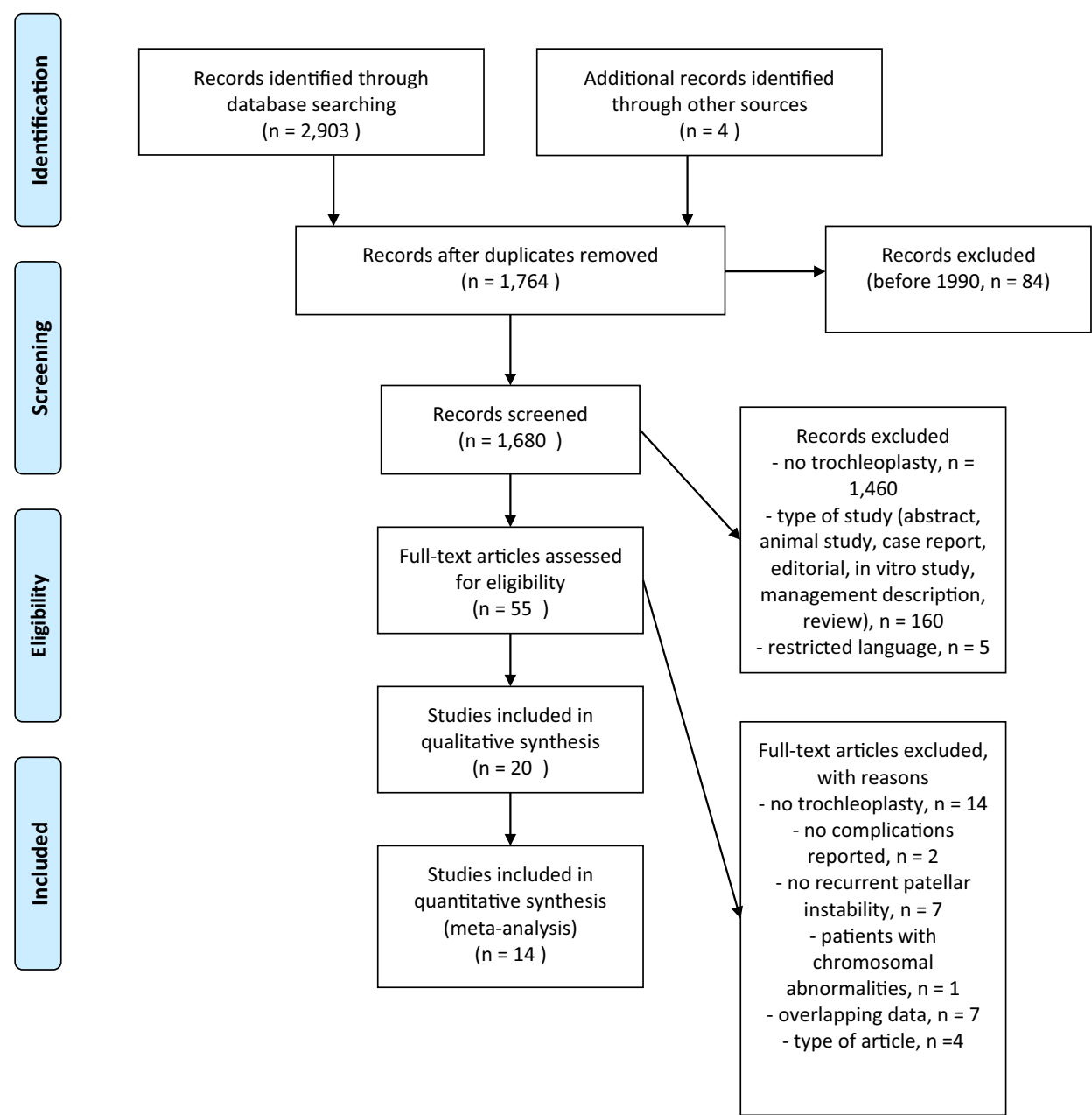

an arthroscopic technique [7], and one on a 'modified' technique [38]. A recession wedge trochleoplasty was reported by one study [33].

\section{Complications and miscellaneous results of techniques included in the meta-analysis}

Meta-analysis could be performed for the complications recurrence of patellar instability (subluxations), recurrent dislocation, PF OA, and further surgery needed for the Dejour and Bereiter trochleoplasty techniques only. Metaanalysis for loss of ROM could only be performed for the Bereiter trochleoplasty. Figures 2, 3, 4 and 5 show the results of the meta-analyses, including proportion of patients with recurrent dislocation (Fig. 2), recurrent instability (Fig. 3), PF OA (Fig. 4) and need for further surgery (Fig. 5). The indications for further surgery were not included in the metaanalysis. For the Bereiter trochleoplasty these were medial subluxation in one patient, reduced ROM in six patients, persistent pain in three patients and recurrence of instability in three patients. For the Dejour trochleoplasty these numbers were complaints of crepitus in two patients, recurrence of instability in ten patients, reduced ROM in 24 patients, persistent pain in one patient, PF OA in six patients, loose absorbable screw heads in two patients, hardware breakage in two patients and a trochlear notch osteophyte in one patient. The proportion of patients with loss of ROM which needed intervention is shown in Fig. 6.

Included in the major complications but not included in the meta-analysis is a pulmonary embolus in one patient in the study of McNamara et al. [19] Minor complications not included in the meta-analysis are a superficial wound infection in two patients in the study of Utting [34] and in four patients in the study of McNamara [19], a deep venous thrombosis in two patients [19, 24], a complication related to anesthesia in two patients [14, 34], a wound healing problem 


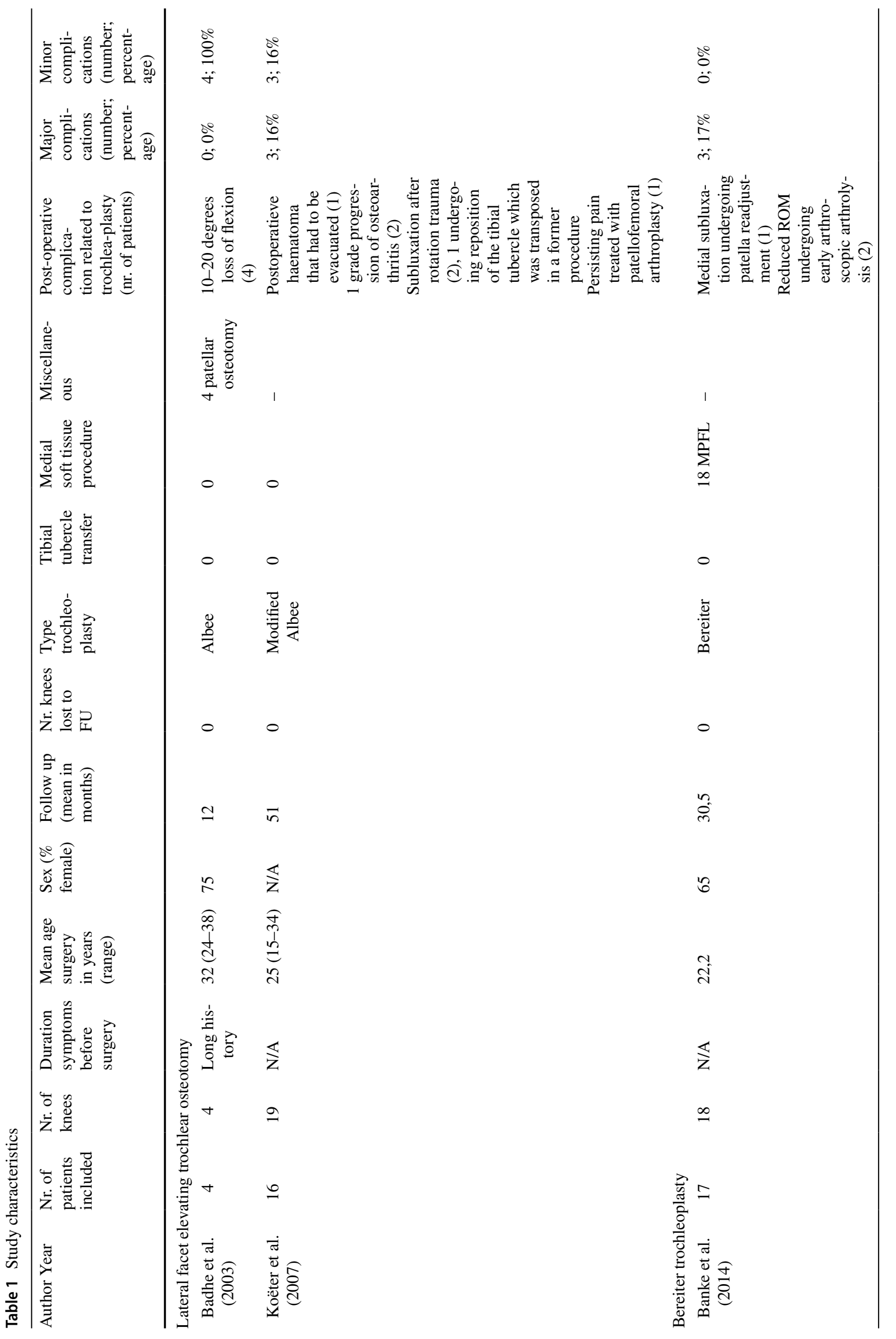




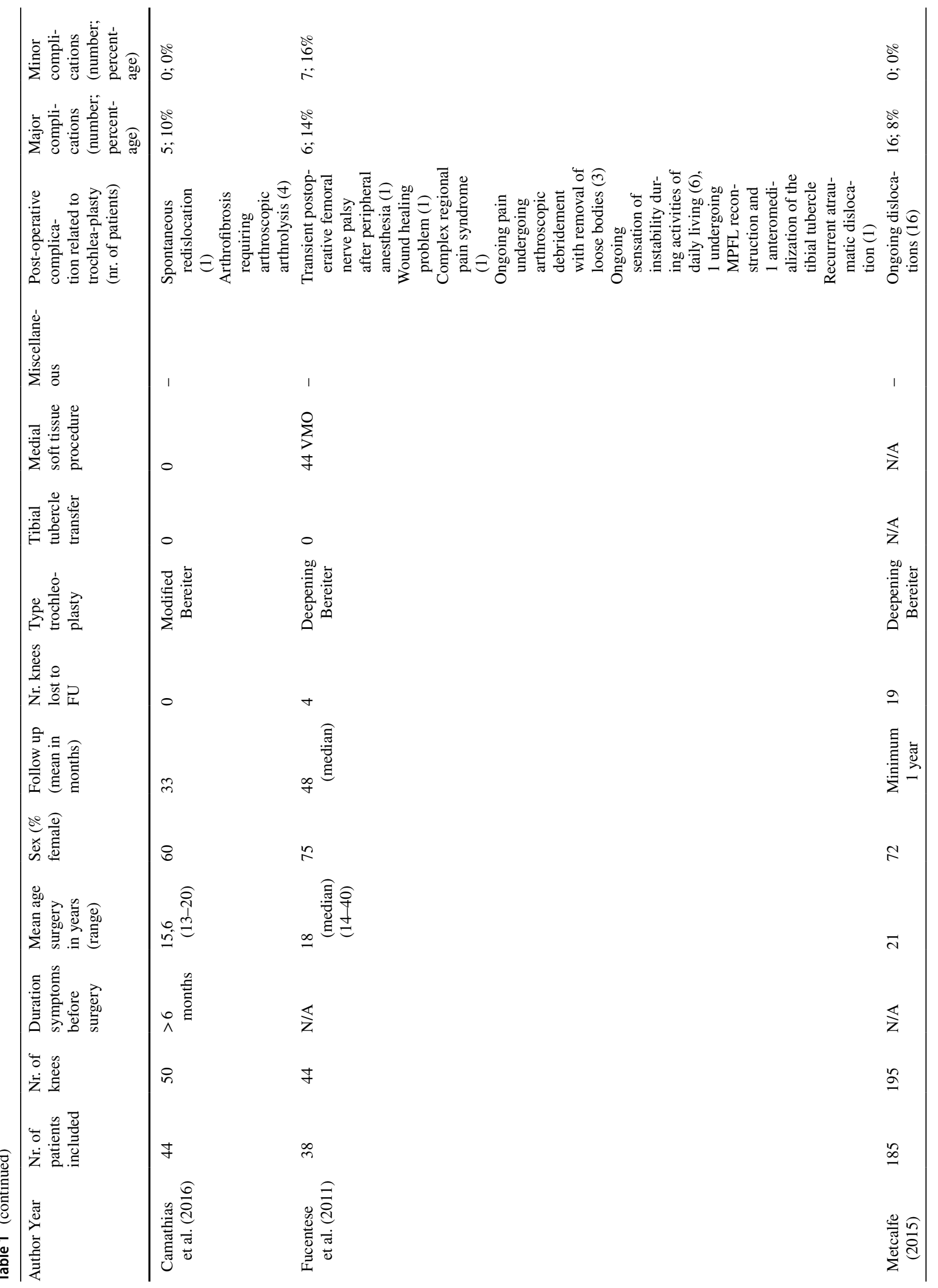


Knee Surgery, Sports Traumatology, Arthroscopy (2018) 26:2841-2857

2847

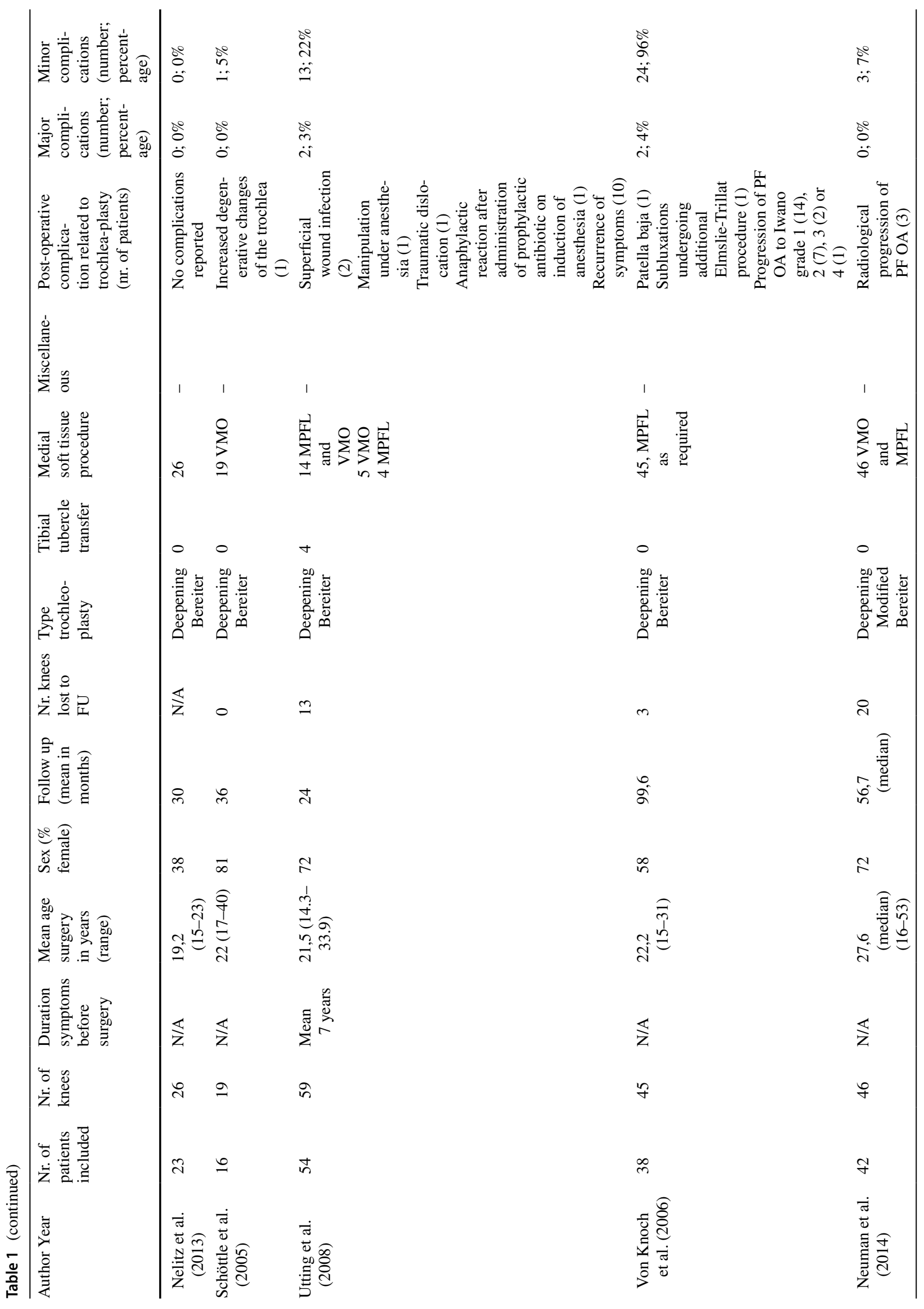

Springer 


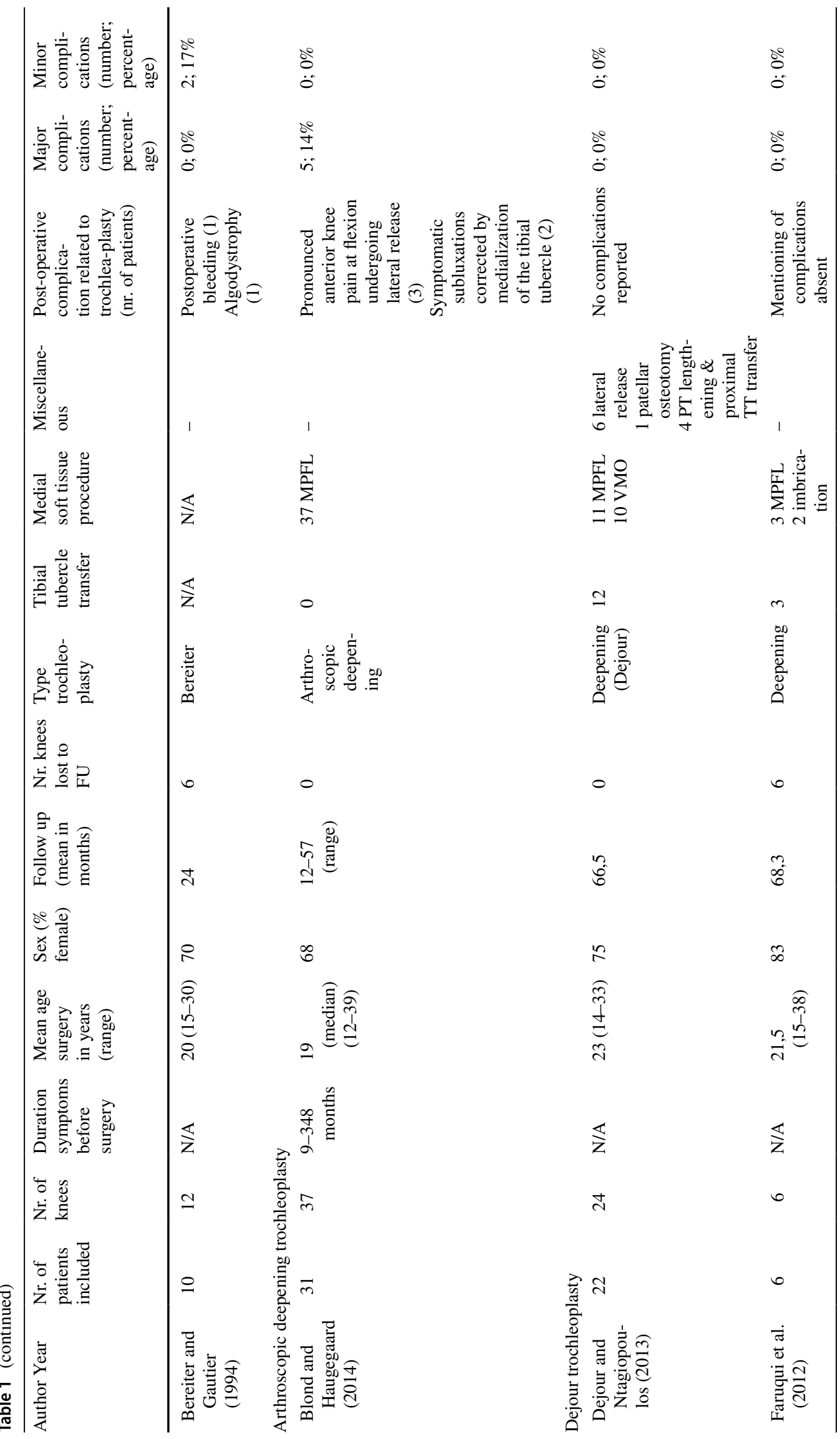




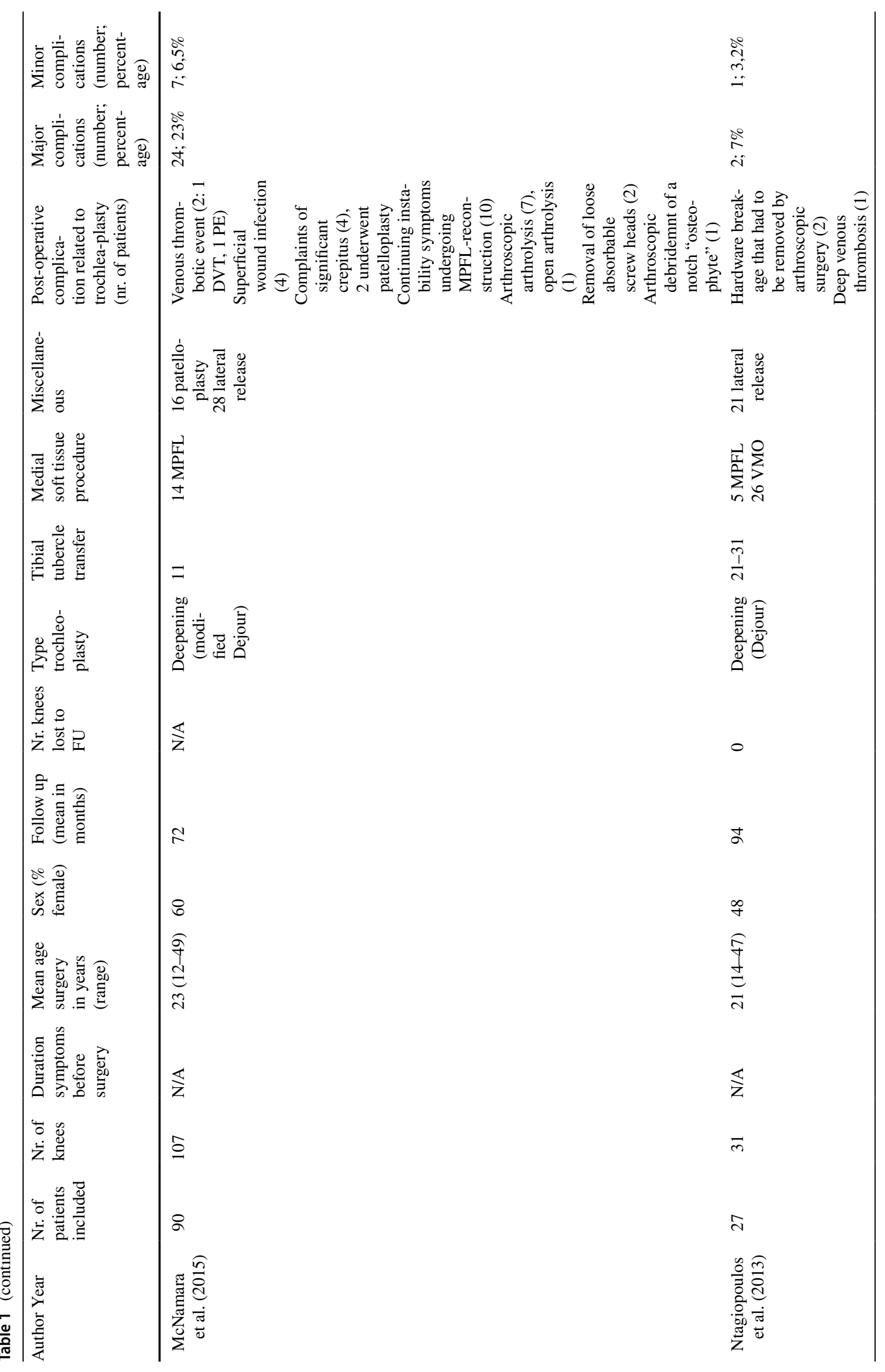




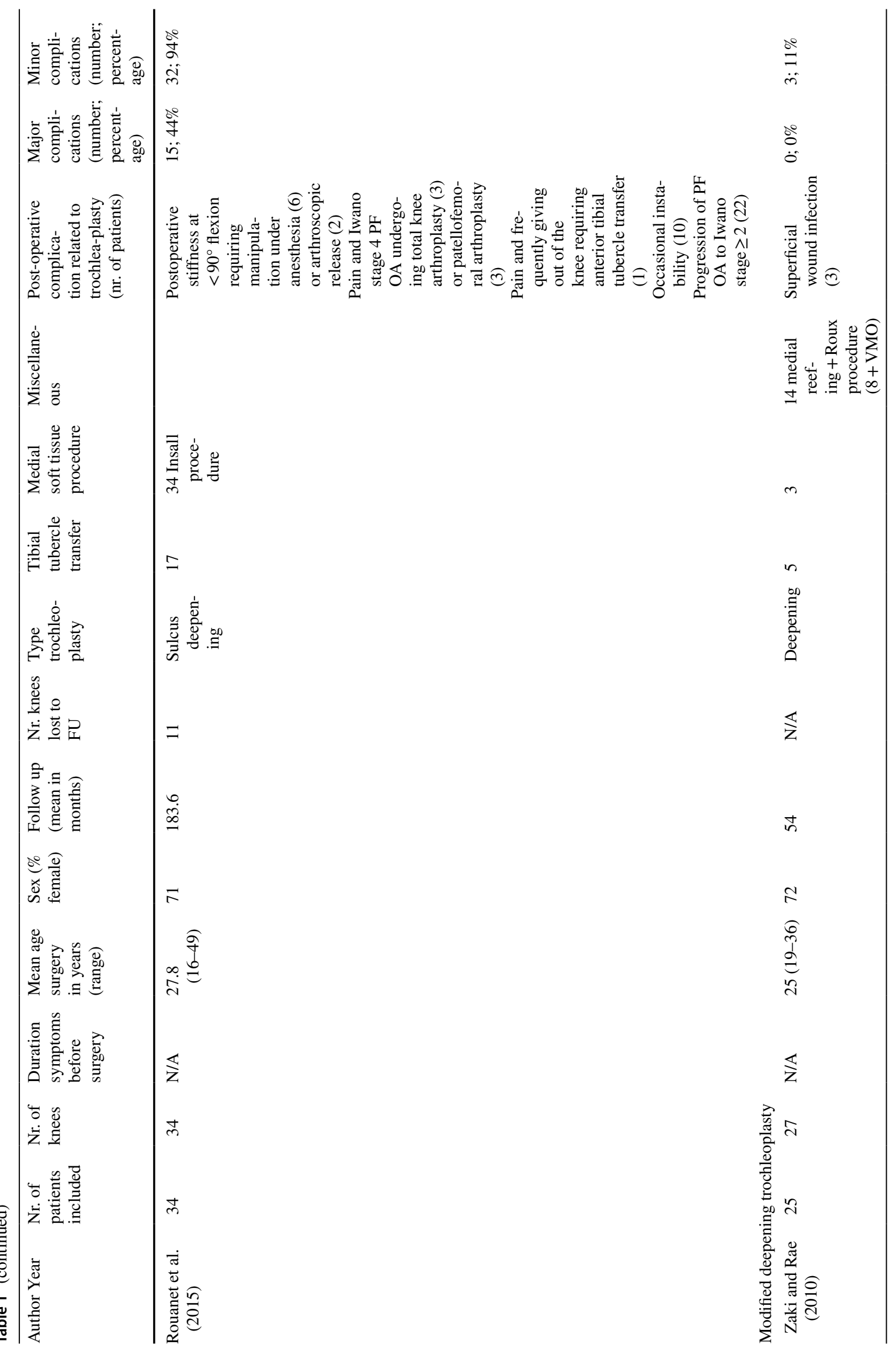




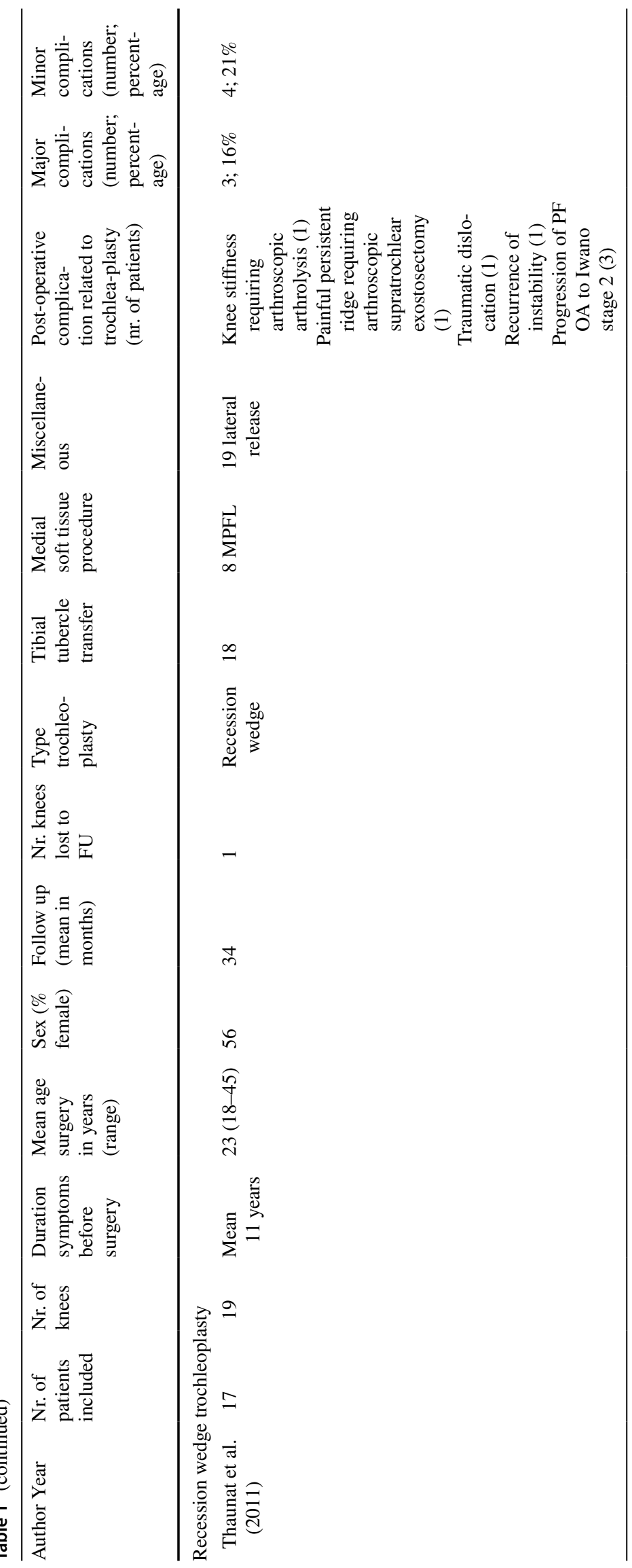




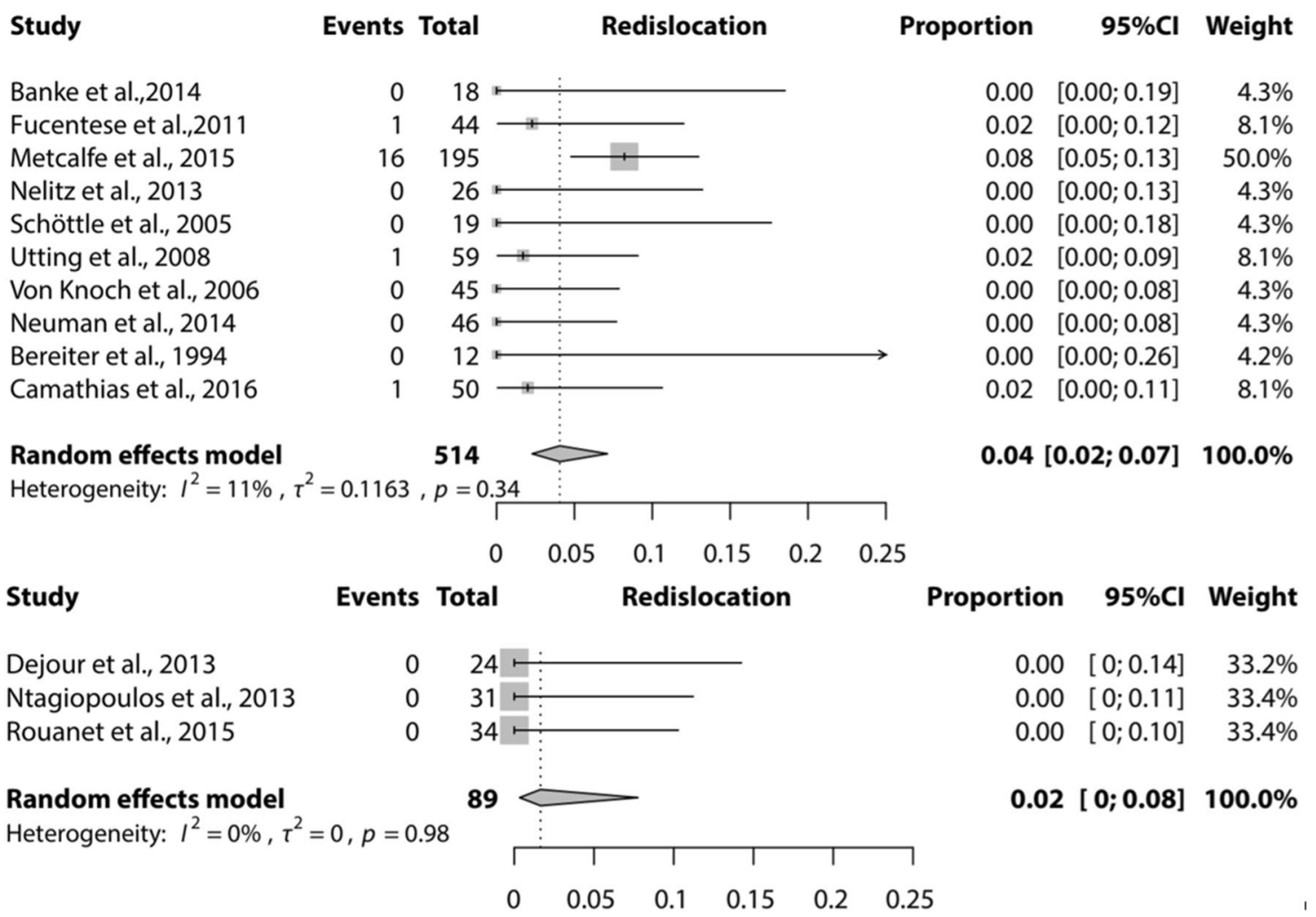

Fig. 2 Forest plot of proportion of recurrent dislocation after a Bereiter trochleoplasty (upper) and Dejour trochleoplasty (lower)

in one patient [14], a complex regional pain syndrome in two patients $[6,14]$ and a postoperative bleeding in one patient [6]. Fifty-eight patients had unchanged or increased pain not requiring reoperation, 95 patients have some residual symptoms such as clicking, swelling or pain. Six patients kept complaints of crepitus without further surgical treatment and 14 patients kept complaints of swelling.

\section{Complications and miscellaneous results of techniques not included in the meta-analysis}

Two studies reported on a lateral facet elevating trochlear osteotomy, one study reported an arthroscopic deepening trochleoplasty, one a modified deepening trochleoplasty, and one a recession wedge trochleoplasty (Table 1).

\section{Discussion}

The most important finding of this study was that Bereiter and Dejour trochleoplasty procedures show complication rates similar to other patellar stabilizing procedures. The rates of reoperation after a Bereiter and Dejour trochleoplasty [0.08 (95\% CI 0.04; 0.14) and 0.20 (95\% CI 0.11; $0.32)]$ are comparable with those found in other systematic reviews of patellar stabilizing procedures $(4.1 \%$ after
MPFL-reconstruction [30], 18\% after tibial tubercle osteotomy [27] and 25\% after trochleoplasty versus $7 \%$ after MPFL-reconstruction [32]). Decreased range of motion and recurrence of instability were the two most frequent reasons for further surgery. The study of McNamara et al. [19] largely contributed (23 patients) to the number of patients returning to the OR after a Dejour trochleoplasty. Ten of these patients underwent an additional MPFL reconstruction and eight underwent arthrolysis. Seven of the eight patients undergoing arthrolysis were from their early cohort of patients before the continuous passive motion was introduced. This study of McNamara et al. might, therefore, confound the rate of reoperation after a Dejour trochleoplasty.

The proportion of recurrent dislocation after a Bereiter or Dejour trochleoplasty [0.04 (95\% CI 0.02-0.07) and $0.02(95 \%$ CI $0-0.08)$ ] was lower than or equal with previous results in literature $[4,26,30]$. In their systematic review, Smith et al. [31] found $13 \%$ recurrent patellar dislocations after 2-5 years follow-up after surgical intervention for patellar dislocation. Meta-analysis showed that the proportion of recurrence of instability (sensation of instability or subluxation) was low for Bereiter [0.06 (95\% CI 0.02-0.13)] and Dejour [0.09 (95\% CI 0.03-0.27)] trochleoplasty. This is low compared with the natural course after patellar dislocation, or patients treated nonsurgically being up to $24 \%$ according to Smith et al. [2, 
Study

Banke et al.,2014

Fucentese et al.,2011

Nelitz et al., 2013

Utting et al., 2008

Von Knoch et al., 2006

Neuman et al., 2014

Bereiter et al., 1994

Camathias et al., 2016

Random effects model

Heterogeneity: $I^{2}=52 \%, \tau^{2}=0.6954, p=0.04$

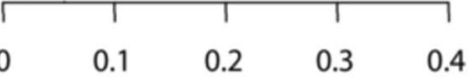

Proportion

$95 \% \mathrm{Cl}$ Weight

$0.00 \quad[0.00 ; 0.19] \quad 7.6 \%$

$0.14 \quad[0.05 ; 0.27] \quad 23.6 \%$

$0.00 \quad[0.00 ; 0.13] \quad 7.7 \%$

$0.17 \quad[0.08 ; 0.29] \quad 25.8 \%$

$0.02 \quad[0.00 ; 0.12] \quad 12.2 \%$

$0.00 \quad[0.00 ; 0.08] \quad 7.7 \%$

$0.00 \quad[0.00 ; 0.26] \quad 7.6 \%$

$0.00 \quad[0.00 ; 0.07] \quad 7.7 \%$

$0.06[0.02 ; 0.13] \quad 100.0 \%$
Study

Dejour et al., 2013

McNamara et al., 2015

Ntagiopoulos et al., 2013

Rouanet et al., 2015

Random effects model

Heterogeneity: $I^{2}=77 \%, \tau^{2}=1.058, p<0.01$

196
Events Total Recurrent patellar instability
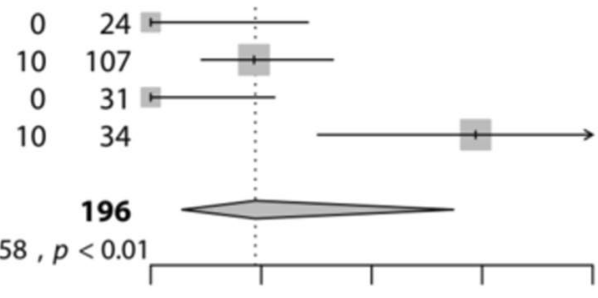

$\begin{array}{ll}0 & 0.1\end{array}$
Proportion

$95 \% \mathrm{Cl}$ Weight

$0.00 \quad[0.00 ; 0.14] \quad 13.8 \%$

$0.09 \quad[0.05 ; 0.17] \quad 36.6 \%$

$0.00 \quad[0.00 ; 0.11] \quad 13.9 \%$

$0.29[0.15 ; 0.47] \quad 35.7 \%$

$0.09[0.03 ; 0.27] \quad 100.0 \%$

Fig. 3 Forest plot of proportion of recurrent patellar instability after a Bereiter trochleoplasty (upper) and Dejour trochleoplasty (lower)

\begin{tabular}{|c|c|c|c|c|c|c|c|c|c|c|}
\hline Study & Events & \multicolumn{2}{|l|}{ Total } & \multicolumn{3}{|c|}{ PFOA } & \multicolumn{2}{|r|}{ Proportion } & $95 \% \mathrm{Cl}$ & Weight \\
\hline Banke et al.,2014 & 0 & $18 \stackrel{\vdots}{\vdots}$ & & & & & & 0.00 & {$[0.00 ; 0.19]$} & $11.8 \%$ \\
\hline Schöttle et al., 2005 & 1 & $19 \div$ & & & & & & 0.05 & {$[0.00 ; 0.26]$} & $17.6 \%$ \\
\hline Von Knoch et al., 2006 & 10 & $45 \vdots \vdots$ & 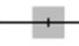 & & & & & 0.22 & {$[0.11 ; 0.37]$} & $32.1 \%$ \\
\hline Neuman et al., 2014 & 3 & 46 & & & & & & 0.07 & {$[0.01 ; 0.18]$} & $26.6 \%$ \\
\hline Camathias et al., 2016 & 0 & $50 \risingdotseq$ & & & & & & 0.00 & {$[0.00 ; 0.07]$} & $11.9 \%$ \\
\hline \multirow{2}{*}{\multicolumn{7}{|c|}{$\begin{array}{ll}\text { Random effects model } & 178 \\
\text { Heterogeneity: } l^{2}=63 \%, \tau^{2}=0.9931, p=0.03\end{array}$}} & \multicolumn{3}{|c|}{$0.07[0.02 ; 0.19]$} & $100.0 \%$ \\
\hline & & & & & & & 7 & & & \\
\hline & & 0 & 0.2 & 0.4 & 0.6 & 0.8 & 1 & & & \\
\hline Study & Events & Total & \multicolumn{4}{|c|}{ PFOA } & & Proportion & $95 \% \mathrm{Cl}$ & Weight \\
\hline Dejour et al., 2013 & 0 & $24 \longmapsto$ & & & & & & 0.00 & {$[0.00 ; 0.14]$} & $31.9 \%$ \\
\hline Ntagiopoulos et al., 2013 & 0 & $31 \longmapsto$ & & & & & & 0.00 & {$[0.00 ; 0.11]$} & $31.9 \%$ \\
\hline Rouanet et al., 2015 & 28 & 34 & & & & + & & 0.82 & {$[0.65 ; 0.93]$} & $36.2 \%$ \\
\hline \multirow{3}{*}{\multicolumn{7}{|c|}{$\begin{array}{l}\text { Random effects model } \\
\text { Heterogeneity: } I^{2}=92 \%, \tau^{2}=13.56, p<0.01\end{array}$}} & \multirow{4}{*}{\multicolumn{3}{|c|}{$0.12[0.00 ; 0.91]$}} & $100.0 \%$ \\
\hline & & & & & & & & & & \\
\hline & & & & & & & & & & \\
\hline & & 0 & 0.2 & 0.4 & 0.6 & 0.8 & & & & \\
\hline
\end{tabular}

Fig. 4 Forest plot of proportion of patellofemoral osteoarthritis (PF OA) after a Bereiter trochleoplasty (upper) and Dejour trochleoplasty (lower) 


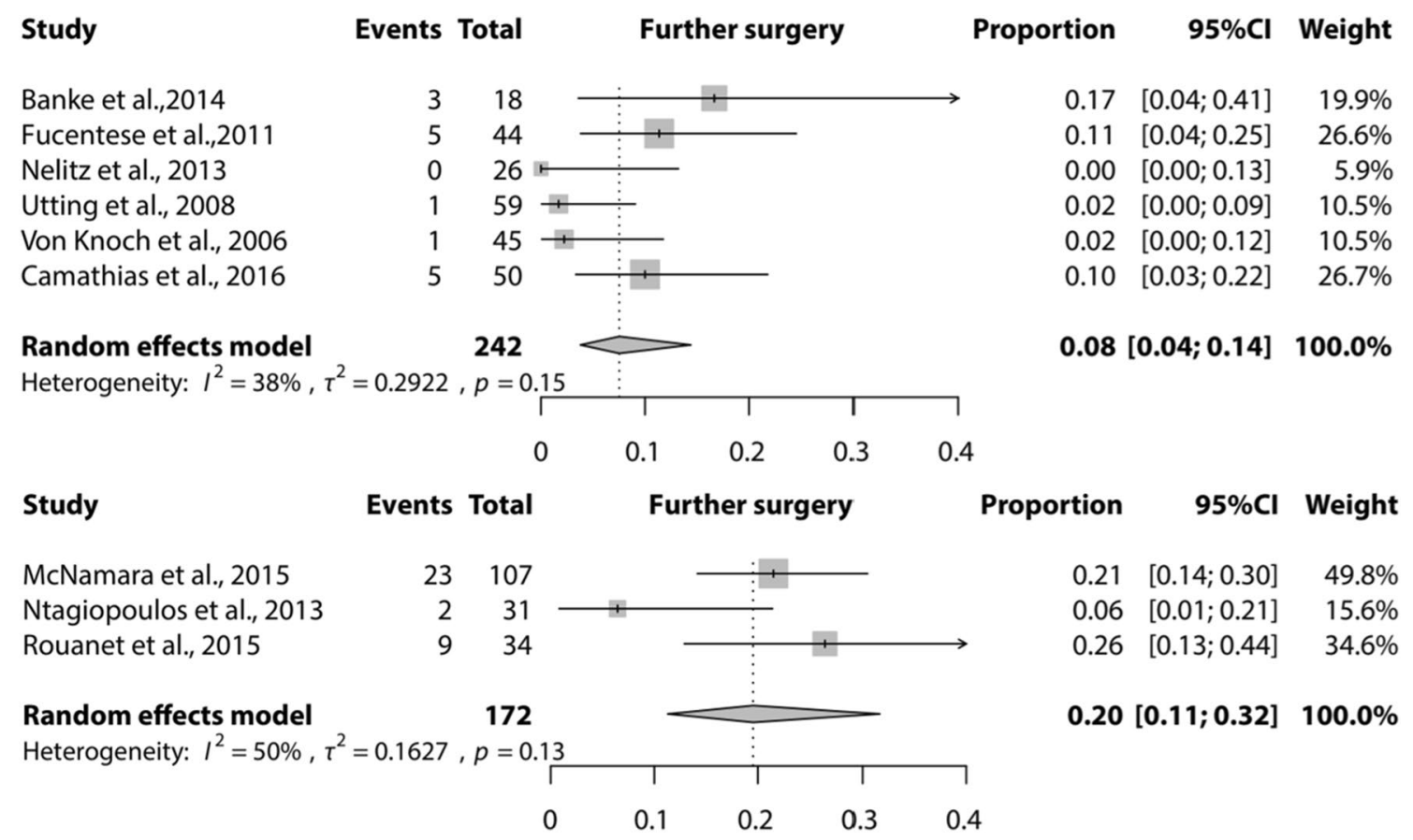

Fig. 5 Forest plot of proportion of patients who needed further surgery after a Bereiter trochleoplasty (upper) and Dejour trochleoplasty (lower)

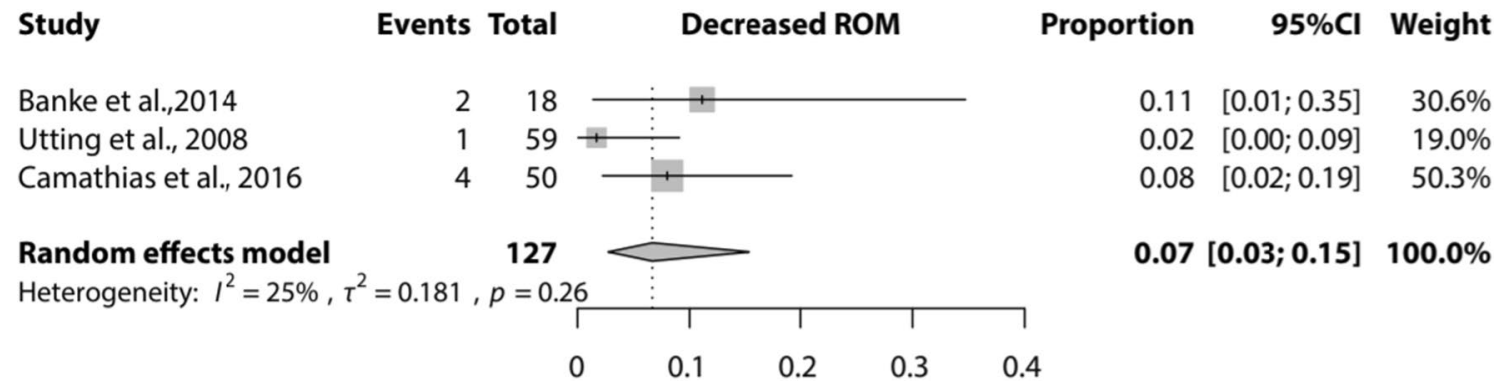

Fig. 6 Forest plot of proportion of patients with loss of range of motion after a Bereiter trochleoplasty

$13,31]$. From these results, it could be hypothesized that these two trochleoplasty techniques are successful in preventing recurrent dislocation and/or instability symptoms, also compared with other surgical interventions.

Seven studies did not report about the presence of PF OA. The rate of development of PF OA would probably increase at longer follow-up, as the development and progression of PF OA in these patients depends on multiple factors, not only a stable patella. Registration of patellofemoral osteoarthritic changes on imaging does not mean that patients have complaints related to PF OA. The number of PF OA should be interpreted as an objective outcome measure and not as a clinically relevant outcome measure if it is asymptomatic. Most of the studies included in this review were not designed to detect PF OA as an outcome measure. The proportion presented in our results could be an underestimation of the true incidence of PF $\mathrm{OA}$ and should be interpreted with caution.

Rare complications that were reported include medial subluxation [5], patella baja [35] and venous thrombotic events $[19,24]$, none were catastrophic. There was no mortality associated with trochleoplasty. One should be aware that these and potential other rare complications can occur after a trochleoplasty since it is a very complex procedure.

Some potential limitations of this study have to be discussed. Since no comparative studies are included, no direct comparison between different techniques could be made. No conclusion can be drawn as to whether one of the techniques is superior to the other in terms of complications of surgery. Furthermore, there is no clear consensus on the indication for trochleoplasty surgery, which makes a direct comparison between studies and/or techniques very difficult. The 
presented complications for different techniques should be interpreted in the context of the individual studies that have been published, including exact indication for surgery, duration and severity of symptoms, and patient factors.

The definition of complications is always arguable and will differ between different clinicians and patients. Mild residual symptoms such as pain, swelling or clicking were classified as an outcome of surgery and not as a complication of surgery. Some complications cannot be definitely assigned to either the trochleoplasty or the additional procedure, this introduces most likely some bias in complication rate.

It should be noted that the absence of complications does not mean that a patient is free of complaints. The rate of complications found in this review is acceptable, but trochleoplasty is still a rather radical surgical procedure with significant risks.

Almost all studies were retrospective or prospective case series. None of the studies were randomized or described a difference between two cohorts. Because of this lack of methodological quality, we did not perform a quality assessment; all studies were regarded low-level evidence.

Publication bias may be present since "negative" results of case series of surgical procedures are less likely to be submitted for publication. Measurement bias may have occurred due to the failure of thorough administration of complications, especially for minor complications in retrospective studies also due to diligence and increased awareness of the screening resulting in higher report of complications.

There might also be sampling bias, since most surgeons who performed trochleoplasty in the articles in this review were experienced surgeons, thus the number of complications might be an underestimation of the true number.

With the limited high-quality evidence available, we think the results of this study a sufficiently accurate represent the complication rate after trochleoplasty procedures including any additional procedures.

\section{Conclusions}

This systematic review and meta-analysis demonstrates that the complications after a Bereiter and Dejour trochleoplasty including additional procedures are in the range of those of other patellar stabilizing procedures. For four other techniques, no meta-analysis could be performed.

Acknowledgements We thank Alice Tillema, Medical Information Specialist, Medical Library, Radboud university medical center, Nijmegen, The Netherlands, for her help during the development of the search strategy.

Author contributions JvS, SvdG, NV and GH designed the study and drafted the research protocol. JvS and SvdG performed the literature search, selection, data extraction and drafted the manuscript. JvS and
GH developed the search strategy and performed the data analysis. JvS, SvdG, NV and GH contributed in the interpretation of the data. SvdG gave final approval of the version to be submitted.

\section{Compliance with ethical standards}

Conflict of interest The authors declare that they have no conflict of interest.

Funding No external source of funding was used.

Ethical approval No ethical approval was obtained because this study was a systematic review with meta-analysis using anonymized data from other published cohort studies.

Informed consent For this type of study informed consent is not required.

Open Access This article is distributed under the terms of the Creative Commons Attribution 4.0 International License (http://creativecommons.org/licenses/by/4.0/), which permits unrestricted use, distribution, and reproduction in any medium, provided you give appropriate credit to the original author(s) and the source, provide a link to the Creative Commons license, and indicate if changes were made.

\section{Appendix}

\section{Pubmed search}

((“Patellar Dislocation"[Mesh] OR ("Patella"[Mesh] AND "Dislocations"[Mesh]) OR ((patella*[tw] OR patello*[tw] OR trochlea*[tw]) AND (dislocat*[tw] OR instability[tw] OR instabilities[tw] OR instable[tw] OR luxation*[tw] OR subluxation*[tw]))) AND (trochleo*[tw] OR trochlea*[tw] OR Sulcus[tw] OR patellar groove[tw] OR patellar dislocation/surgery)) OR patellar dislocation/complications.

\section{Embase and Web of Science search terms}

((Patella dislocation/OR (exp patella/AND exp dislocation)) OR ((patella* or patella* or trochlea) AND (dislocate* or instability or instabilities or instable or luxation* or subluxation*))ti.ab.kw.) AND ((trochlea* or trochleo*).ti.ab.kw. OR sulcus ti.ab.kw. OR patella dislocation/surgery) OR patella dislocation/complication. Limits: conference abstract or conference proceeding.

\section{Cochrane Library search}

Patellar Dislocation [MeSH] OR (Patella [MeSH] AND Dislocations $[\mathrm{MeSH}])$ OR ((patella* or patella* or trochlea) AND (dislocate* or instability or instabilities or instable or luxation* or subluxation*)). 


\section{References}

1. Albee (1915) The bone graft wedge in the treatment of habitual dislocation of the patella. Med Rec 88:257-259

2. Arnbjörnsson A EN, Rydling O, Stockerup R, Ryd L (1992) The natural history of recurrent dislocation of the patella. J Bone $\mathrm{Jt}$ Surg Br 74:140-142

3. Badhe NP, Forster IW (2003) Patellar osteotomy and Albee's procedure for dysplastic patellar instability. Eur J Orthop Surg Traumatol 13:43-47

4. Balcarek P, Rehn S, Howells NR, Eldridge JD, Kita K, Dejour D et al (2016) Results of medial patellofemoral ligament reconstruction compared with trochleoplasty plus individual extensor apparatus balancing in patellar instability caused by severe trochlear dysplasia: a systematic review and metaanalysis. Knee Surg Sports Traumatol Arthrosc. doi:10.1007/ s00167-016-4365-x

5. Banke IJ, Kohn LM, Meidinger G, Otto A, Hensler D, Beitzel K et al (2014) Combined trochleoplasty and MPFL reconstruction for treatment of chronic patellofemoral instability: a prospective minimum 2-year follow-up study. Knee Surg Sports Traumatol Arthrosc 22:2591-2598

6. Bereiter H GE (1994) Die Trochleaplastik als chirurgische Therapie der rezidivierenden Patellaluxation bei Trochleadysplasie des Femurs. Arthroskopie 7:281-286

7. Blond L, Haugegaard M (2014) Combined arthroscopic deepening trochleoplasty and reconstruction of the medial patellofemoral ligament for patients with recurrent patella dislocation and trochlear dysplasia. Knee Surg Sports Traumatol Arthrosc 22:2484-2490

8. Camathias C, Studer K, Kiapour A, Rutz E, Vavken P (2016) Trochleoplasty as a solitary treatment for recurrent patellar dislocation results in good clinical outcome in adolescents. Am J Sports Med 44:2855-2863

9. Dejour D, Byn P, Ntagiopoulos PG (2013) The Lyon's sulcusdeepening trochleoplasty in previous unsuccessful patellofemoral surgery. Int Orthop 37:433-439

10. Dejour D, Le Coultre B (2007) Osteotomie in patello-femoral instabilities. Sports Med Arthrosc Rev 15:39-46

11. Dejour H, Walch G, Neyret P, Adeleine P (1990) [Dysplasia of the femoral trochlea]. Rev Chir Orthop Reparatrice Appar Mot $76: 45-54$

12. Faruqui S, Bollier M, Wolf B, Amendola N (2012) Outcomes after trochleoplasty. Iowa Orthop J 32:196-206

13. Fithian DC, Paxton EW, Stone ML, Silva P, Davis DK, Elias DA et al (2004) Epidemiology and natural history of acute patellar dislocation. Am J Sports Med 32:1114-1121

14. Fucentese SF, Zingg PO, Schmitt J, Pfirrmann CWA, Meyer DC, Koch PP (2011) Classification of trochlear dysplasia as predictor of clinical outcome after trochleoplasty. Knee Surg Sports Traumatol Arthrose 19:1655-1661

15. Goutallier D, Raou D, Van Driessche S (2002) Retro-trochlear wedge reduction trochleoplasty for the treatment of painful patella syndrome with protruding trochleae. Technical note and early results. Rev Chir Orthop Reparatrice Appar Mot 88:678-685

16. Koeter S, Pakvis D, Van Loon CJM, Van Kampen A (2007) Trochlear osteotomy for patellar instability: satisfactory minimum 2-year results in patients with dysplasia of the trochlea. Knee Surg Sports Traumatol Arthrosc 15:228-232

17. Longo UG, Vincenzo C, Mannering N, Ciuffreda M, Salvatore G, Berton A et al. (2017) Trochleoplasty techniques provide good clinical results in patients with trochlear dysplasia. Knee Surg Sports Traumatol Arthrosc. doi:10.1007/s00167-017-4584-9

18. Masse Y (1978) Treatment of dislocations of the patella by deepening the inter condylar groove [French]. Revue de Chirurgie Orthopedique et Reparatrice de l'Appareil Moteur 64:3-17
19. McNamara I, Bua N, Smith TO, Ali K, Donell ST (2015) Deepening trochleoplasty with a thick osteochondral flap for patellar instability: clinical and functional outcomes at a mean 6-year follow-up. Am J Sports Med 43:2706-2713

20. Metcalfe AJ, Clark DA, Kemp M, Eldridge JD (2015) The Bristol experience of the Bereiter trochleoplasty: indications and technique. Oper Tech Sports Med 23:123-128

21. Moher D LA, Tetzlaff J, Altman DG, The PRIMSA Group (2009) Preferred reporting items for systematic reviews and meta-analyses: the PRISMA statement. PLoS Med 6:e1000097

22. Nelitz M, Dreyhaupt J, Lippacher S (2013) Combined trochleoplasty and medial patellofemoral ligament reconstruction for recurrent patellar dislocations in severe trochlear dysplasia: a minimum 2-year follow-up study. Am J Sports Med 41:1005-1012

23. Neumann MV, Stalder M, Schuster AJ (2016) Reconstructive surgery for patellofemoral joint incongruency. Knee Surg Sports Traumatol Arthrosc 24:873-878

24. Ntagiopoulos PG, Byn P, Dejour D (2013) Midterm results of comprehensive surgical reconstruction including sulcus-deepening trochleoplasty in recurrent patellar dislocations with highgrade trochlear dysplasia. Am J Sports Med 41:998-1004

25. Ntagiopoulos PG, Dejour D (2014) Current concepts on trochleoplasty procedures for the surgical treatment of trochlear dysplasia. Knee Surg Sports Traumatol Arthrosc 22:2531-2539

26. Nwachukwu BU, So C, Schairer WW, Green DW, Dodwell ER (2016) Surgical versus conservative management of acute patellar dislocation in children and adolescents: a systematic review. Knee Surg Sports Traumatol Arthrosc 24:760-767

27. Payne J, Rimmke N, Schmitt LC, Flanigan DC, Magnussen RA (2015) The incidence of complications of tibial tubercle osteotomy: a systematic review. Arthroscopy 31:1819-1825

28. Rouanet T, Gougeon F, Fayard JM, Remy F, Migaud H, Pasquier G (2015) Sulcus deepening trochleoplasty for patellofemoral instability: a series of 34 cases after 15 years postoperative followup. Orthop Traumatol Surg Res 101:443-447

29. Schottle PB, Fucentese SF, Pfirrmann C, Bereiter H, Romero J (2005) Trochleaplasty for patellar instability due to trochlear dysplasia: a minimum 2-year clinical and radiological follow-up of 19 knees. Acta Orthop Scand 76:693-698

30. Shah JN, Howard JS, Flanigan DC, Brophy RH, Carey JL, Lattermann C (2012) A systematic review of complications and failures associated with medial patellofemoral ligament reconstruction for recurrent patellar dislocation. Am J Sports Med 40:1916-1923

31. Smith T, Donell S, Song F, Hing CB (2015) Surgical versus nonsurgical interventions for treating patellar dislocation. Cochrane Database Syst Rev 26:CD008106

32. Testa EA, Camathias C, Amsler F, Henle P, Friederich NF, Hirschmann MT (2015) Surgical treatment of patellofemoral instability using trochleoplasty or MPFL reconstruction: a systematic review. Knee Surg Sports Traumatol Arthrosc 25:2309-2320

33. Thaunat M, Bessiere C, Pujol N, Boisrenoult P, Beaufils P (2011) Recession wedge trochleoplasty as an additional procedure in the surgical treatment of patellar instability with major trochlear dysplasia: early results. Orthop Traumatol Surg Res 97:833-845

34. Utting MR, Mulford JS, Eldridge JDJ (2008) A prospective evaluation of trochleoplasty for the treatment of patellofemoral dislocation and instability. J Bone Jt Surg Br 90:180-185

35. von Knoch F, Bohm T, Burgi ML, von Knoch M, Bereiter H (2006) Trochleaplasty for recurrent patellar dislocation in association with trochlear dysplasia: a 4- to 14-year follow-up study. J Bone Jt Surg Br 88:1331-1335

36. Weber AE, Nathani A, Dines JS, Allen AA, Shubin-Stein BE, Arendt EA et al (2016) An algorithmic approach to the management of recurrent lateral patellar dislocation. J Bone Jt Surg Am 98:417-427 
37. Weinberger JM, Fabricant PD, Taylor SA, Mei JY, Jones KJ (2017) Influence of graft source and configuration on revision rate and patient-reported outcomes after MPFL reconstruction: a systematic review and meta-analysis. Knee Surg Sports Traumatol Arthrosc 25:2511-2519
38. Zaki SH, Rae PJ (2010) Femoral trochleoplasty for recurrent patellar instability: a modified surgical technique and its medium-term results. Curr Orthop Pract 21:153-157 